\title{
Long-Term Efficacy and Safety of Brexpiprazole in Elderly Japanese Patients with Schizophrenia: A Subgroup Analysis of an Open-Label Study
}

This article was published in the following Dove Press journal: Neuropsychiatric Disease and Treatment

\section{Ken Inada (D) \\ Sakiko Yamada $\mathbb{D}^{2}$ \\ Hisashi Akiyoshi \\ Yoshitsugu Kojima ${ }^{2}$ \\ Shuichi Iwashita $\mathbb{D D}^{3}$ \\ Jun Ishigooka ${ }^{4}$}

'Department of Psychiatry, Tokyo Women's Medical University, Tokyo, Japan; ${ }^{2}$ Department of Medical Affairs, Otsuka Pharmaceutical Co., Ltd., Tokyo, Japan; ${ }^{3}$ Headquarters of Clinical Development, Otsuka Pharmaceutical Co., Ltd., Osaka, Japan; ${ }^{4}$ Institute of CNS Pharmacology, Tokyo, Japan
Correspondence: Sakiko Yamada Department of Medical Affairs, Otsuka Pharmaceutical Co, Ltd., Shinagawa Grand Central Tower, 2-6-14 Konan, Minato-Ku, Tokyo 108-8242, Japan

Tel +8I-3-67I7-| 400

Fax +8I-3-67I7-1398

Email yamadasa@otsuka.jp
Purpose: This study was performed to assess the long-term efficacy, safety, and tolerability of brexpiprazole in elderly Japanese patients with schizophrenia.

Methods: This is a post hoc analysis of a previous open-label study conducted over 56 weeks which consisted of two consecutive phases: a 4-week switching period and a 52-week open-label period. Mean change in the Positive and Negative Syndrome Scale (PANSS) total score, response rates, number and incidence of treatment-emergent adverse events (TEAEs), and other safety parameters were analyzed using descriptive statistics based on age group (elderly, $\geq 65$ and non-elderly, $<65$ ).

Results: This post hoc analysis included 208 de novo patients of which 33 were elderly. The continuation rate in elderly patients was $54.5 \%$, and the mean daily dose and treatment duration of brexpiprazole in elderly patients at week 56 were similar to those of non-elderly patients. The mean change in the PANSS total score from the baseline to week 56 was -13.8 in elderly patients and this improvement was maintained throughout the open-label phase. This outcome was comparable to that of the non-elderly patients $(-9.0)$. The incidence rate of TEAEs was $97.0 \%$ in elderly patients and $82.3 \%$ in non-elderly patients. Most of the TEAEs were either mild $(75.8 \%)$ or moderate $(18.2 \%)$ in severity in the elderly patients and the incidence of TEAEs leading to discontinuation was lower in elderly (9.1\%) than in nonelderly patients $(13.1 \%)$. The most commonly observed adverse events in elderly patients were nasopharyngitis $(30.3 \%)$ and worsening of schizophrenia $(27.3 \%)$. The safety profiles in both groups were similar.

Conclusion: Brexpiprazole was shown to be safe and effective in the treatment of elderly Japanese patients with schizophrenia.

Keywords: brexpiprazole, long-term, schizophrenia, elderly, safety, Japanese

\section{Introduction}

Schizophrenia is a serious, chronic mental disorder, in which disease onset occurs during adolescence to young adulthood and requires life-long treatment. It was reported in 2017 that approximately 20 million people were affected worldwide and the global burden associated with schizophrenia remains large. ${ }^{1}$ In recent years, the number of elderly patients with schizophrenia has been increasing in Japan as the population progressively ages. According to the patient survey conducted in Japan in 2017 by Ministry of Health, Labour and Welfare, the number of elderly patients (aged 65 years or older) with schizophrenia were around 224,000, representing about $28 \%$ of all patients with schizophrenia. 
Generally, low doses of antipsychotics are recommended for the treatment of elderly patients because they tend to have adverse events more frequently than nonelderly patients, ${ }^{2}$ probably due to a decline in physiological functions and physical complications. However, there have only been few reports that examined appropriate doses of antipsychotics and adverse events in elderly patients with schizophrenia.

Brexpiprazole has been approved for the treatment of schizophrenia in the US, Canada, the EU and some Asian countries, including Japan, and of major depressive disorder as an adjunctive therapy to antidepressants in the US and Canada. ${ }^{3,4}$ Brexpiprazole is a serotonin-dopamine activity modulator that acts as a partial agonist at serotonin 5- $\mathrm{HT}_{1 \mathrm{~A}}$ and dopamine $\mathrm{D}_{2}$ receptors, and an antagonist at serotonin 5- $\mathrm{HT}_{2 \mathrm{~A}}$ receptors. ${ }^{5,6}$ Brexpiprazole has demonstrated its efficacy and safety with favorable tolerability in acute patients with schizophrenia in short-term, randomized, double-blind placebo-controlled studies $^{7-9}$ and relapse prevention in a long-term, randomized, doubleblind, placebo-controlled, maintenance study. ${ }^{10}$ In terms of long-term treatment, the results of 52-week, openlabel studies indicated good tolerability with a favorable safety profile. ${ }^{11,12}$ In Japanese patients with schizophrenia, the efficacy and safety of brexpiprazole were demonstrated in acute schizophrenia in a 6-week, randomized, doubleblind, placebo-controlled study ${ }^{9}$ and long-term safety and efficacy were also confirmed in a 52-week, open-label study. ${ }^{13}$ However, only a few reports on brexpiprazole have focused on elderly patients with schizophrenia.

This study was conducted to evaluate the long-term efficacy and safety of brexpiprazole in elderly patients with schizophrenia by analyzing subgroups and comparing the data of elderly and non-elderly patients in a 52-week open-label study. ${ }^{13}$

\section{Methods}

\section{Study Design and Patients}

This was a post hoc subgroup analysis of a multicenter, open-label study conducted in Japan between 2011 and 2015 to investigate the long-term safety and efficacy of brexpiprazole in patients with schizophrenia (ClinicalTrials. gov NCT01456897). ${ }^{13}$ This study consisted of two consecutive phases (total 56 weeks): a 4-week switching period in which patients were switched from their prior antipsychotic therapy to brexpiprazole, and a 52-week open-label treatment period. A full and detailed description of the methods employed in the study, and the inclusion and exclusion criteria can be found in the primary report. ${ }^{13}$

Briefly, only de novo patients were enrolled in this analysis; ie, those who had been diagnosed with schizophrenia based on DSM-IV-TR diagnostic criteria and were 18 years or older, were receiving treatment with oral antipsychotics (other than clozapine), were considered to require maintenance therapy with antipsychotics, and were considered suitable for monotherapy with brexpiprazole. The patients were generally managed on an outpatient basis, but it was permissible to include those who were hospitalized for social issues. Patients presenting with their first episode schizophrenia, or who had severe akathisia, tardive dyskinesia, mental disorders other than schizophrenia, had a history of substance abuse or dependence within the past 180 days, or electroconvulsive treatment 60 days prior to informed consent were excluded. Generally, all use of psychotropics including antipsychotics was prohibited during the open-label treatment period.

The study was conducted in compliance with the Declaration of Helsinki, the Japanese regulatory requirements, and Good Clinical Practice guideline. The protocol was approved by the institutional review board (IRB) at each participating investigational site (Table S1), and written informed consent was obtained from all patients. The first IRB approval was issued by Goryokai Hospital IRB (ID:331-10-003).

Administration of brexpiprazole was started at $1 \mathrm{mg} /$ day during the switching period and the dose of antipsychotics previously being taken was gradually reduced, switching to monotherapy with brexpiprazole at $2 \mathrm{mg} /$ day from week 4 of the switching period (using an addon and taper off method). In the following open-label treatment period, the dose was gradually increased to $4 \mathrm{mg} /$ day in 1-mg increments based on the criteria, provided the investigator determined that there was no tolerability-related problems and the Clinical Global Impression-Improvement (CGI-I) score was 3-7. The dose could be reduced to a minimum of $1 \mathrm{mg}$ /day depending on tolerability.

\section{Assessments and Statistical Analyses}

The efficacy and safety datasets for the entire 56 weeks (4-week switching period and 52-week open-label treatment period) included patients who received one or more doses of brexpiprazole and had one or more efficacy/safety evaluations after the start of brexpiprazole administration. Baseline data were collected before 
Table I Demographic and Baseline Clinical Characteristics

\begin{tabular}{|c|c|c|c|}
\hline & $\begin{array}{l}\text { Elderly } \\
(\geq 65)\end{array}$ & $\begin{array}{l}\text { Non-Elderly } \\
(<65)\end{array}$ & Total \\
\hline & $(N=33)$ & $(N=175)$ & $(\mathrm{N}=208)$ \\
\hline Male, n (\%) & $16(48.5)$ & $81(46.3)$ & $97(46.6)$ \\
\hline Age (years) & $69.5 \pm 4.2$ & $40.8 \pm 11.5$ & $45.4 \pm 15.0$ \\
\hline Age at first diagnosis (years) & $33.7 \pm 14.2$ & $27.6 \pm 9.3$ & $28.5 \pm 10.5$ \\
\hline Duration of illness (years) & $35.8 \pm 13.8$ & $13.2 \pm 10.9$ & $16.8 \pm 14.0$ \\
\hline Body weight $(\mathrm{kg})$ & $55.0 \pm 13.3$ & $66.7 \pm 13.7$ & $64.9 \pm 14.2$ \\
\hline BMI $\left(\mathrm{kg} / \mathrm{m}^{2}\right)$ & $22.7 \pm 5.0$ & $24.8 \pm 4.2$ & $24.5 \pm 4.4$ \\
\hline PANSS total score & $75.4 \pm 25.8$ & $68.9 \pm 21.2$ & $69.9 \pm 22.0$ \\
\hline CGI-S score & $3.88 \pm 1.29$ & $3.34 \pm 1.04 *$ & $3.43 \pm 1.10^{* *}$ \\
\hline Chlorpromazine dose equivalence of prior antipsychotics ( $\mathrm{mg} /$ day) & $521.6 \pm 462.6$ & $478.7 \pm 337.4$ & $485.6 \pm 359.4$ \\
\hline \multicolumn{4}{|l|}{ Prior antipsychotic therapy, n (\%) } \\
\hline Aripiprazole & $7(21.2)$ & $86(49.1)$ & $93(44.7)$ \\
\hline Olanzapine & $13(39.4)$ & $28(16.0)$ & $41(19.7)$ \\
\hline Quetiapine & $4(12.1)$ & $14(8.0)$ & $18(8.7)$ \\
\hline Risperidone & $6(18.2)$ & $32(18.3)$ & $38(18.3)$ \\
\hline
\end{tabular}

Notes: Data, Mean \pm SD (Standard Deviation). $*_{n}=168, *^{*}=201$.

Abbreviations: BMI, body mass index; PANSS, Positive and Negative Syndrome Scale.

initiation of the switching period. The analyses were performed according to age group (elderly, $\geq 65$ years; non-elderly, $<65$ years; and total). Demographic and baseline clinical characteristics were summarized by descriptive statistics of the subgroups, which included age, age at first episode of schizophrenia, duration of schizophrenia, gender, body weight, body mass index (BMI), Positive and Negative Syndrome Scale (PANSS) total score, chlorpromazine dose equivalence of prior antipsychotics and main prior antipsychotics. Prior antipsychotic usage was defined as antipsychotics used 30 days prior to informed consent.

The number of patients who completed or discontinued from the study, reason for discontinuation, and duration and dosage of brexpiprazole were summarized by descriptive statistics for each subgroup. Kaplan-Meier curves for the days from the start to the end of dosing were calculated. Patients who discontinued the study were considered to have an event, and those who completed the study were censored by the number of days to the end date of study drug administration. Efficacy was evaluated based on the PANSS total score and response rate. Response rates at week 56 [defined as $\geq 30 \%$ reduction in PANSS total score from baseline or a CGI-I score of 1 (very much improved) or 2 (much improved) at week 56] were also calculated using OC data. Safety was assessed by treatment-emergent adverse events (TEAEs), severity of TEAEs, serum prolactin level, body weight, and corrected QT interval (QTc) on 12-lead electrocardiogram. Extrapyramidal symptoms (EPS) were assessed using the Drug-Induced Extrapyramidal Symptoms Scale (DIEPSS), Abnormal Involuntary Movement Scale (AIMS), and Barnes Akathisia Rating Scale (BARS). Descriptive statistics of values of the changes in PANSS total score, DIEPSS, AIMS and BARS scores were calculated using observed case (OC) data. Missing data were not imputed.

\section{Results}

\section{Patient Disposition and Characteristics}

In total, 208 de novo patients with schizophrenia were enrolled; 33 patients were elderly and 175 were nonelderly. The baseline demographics and clinical characteristics are shown in Table 1 . The mean age (range) was 69.5 (65-79) years in elderly patients and 40.8 (18-64) years in non-elderly patients. The mean PANSS total score at baseline in elderly patients was higher than that in non-elderly patients (75.4 and 68.9, respectively). The mean dosage of prior antipsychotics (chlorpromazine dose equivalence) was higher in elderly than in non-elderly patients (521.6 and $478.7 \mathrm{mg} /$ day, respectively). The most frequently used prior antipsychotic was olanzapine (39.4\%) in elderly patients and aripiprazole $(49.1 \%)$ in non-elderly patients. 


\section{Treatment Exposure}

During the switching period, 24 patients discontinued the study and 184 patients were enrolled in the open- label treatment period. The completion rate for the entire 56-week treatment period was $54.5 \%$ in elderly patients and $52.0 \%$ in non-elderly patients (Figure 1).

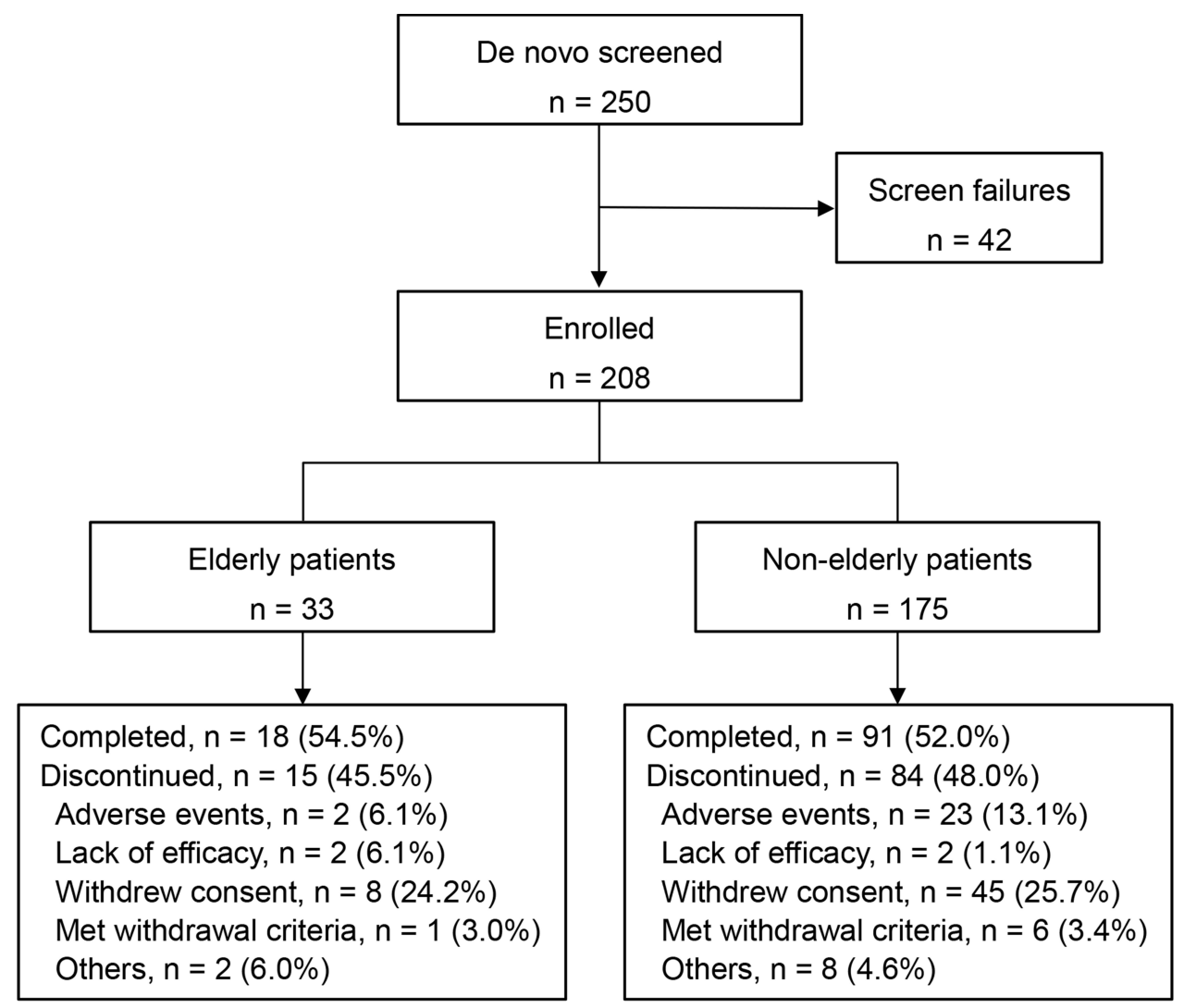

Figure I Patient disposition.

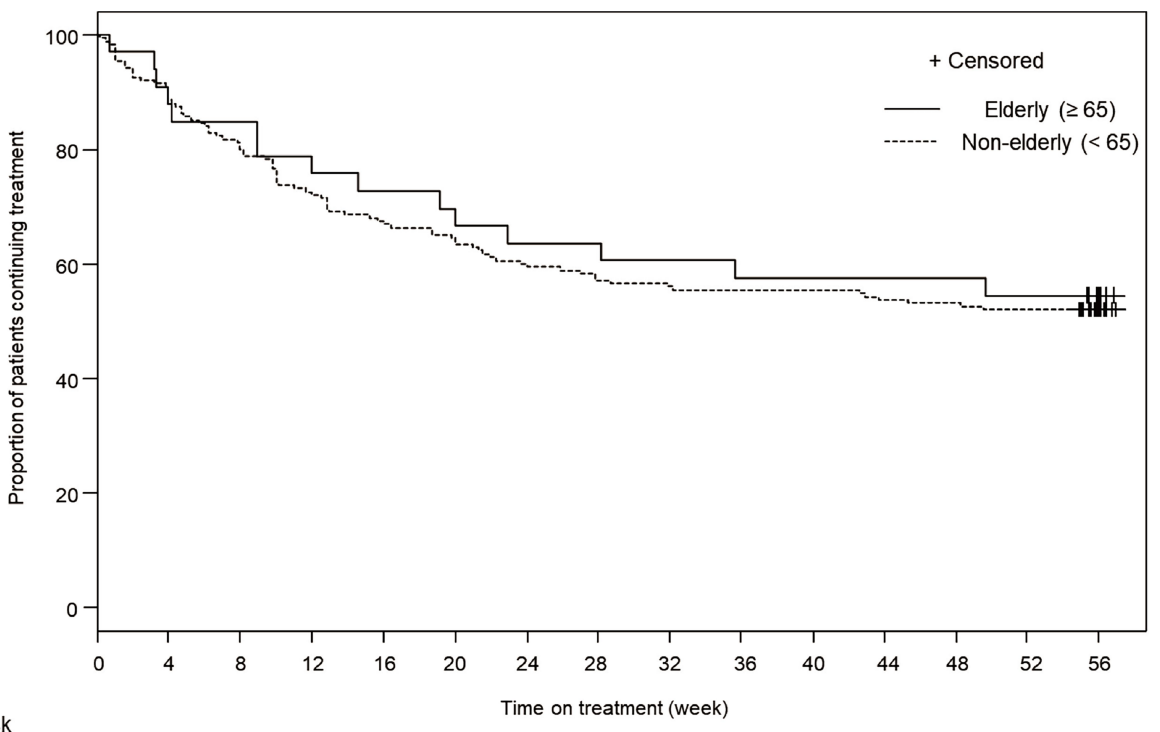

\begin{tabular}{cccccccccccccccc} 
At risk & \multicolumn{10}{c}{ Time on treatment (week) } \\
Elderly $(\geq 65)$ & 33 & 30 & 28 & 26 & 24 & 23 & 21 & 21 & 20 & 19 & 19 & 19 & 19 & 18 & 10 \\
Non-elderly $(<65)$ & 175 & 159 & 142 & 127 & 118 & 113 & 105 & 100 & 98 & 97 & 97 & 94 & 93 & 91 & 60
\end{tabular}

Figure 2 Kaplan-Meier survival curves. 
Table 2 Summary of the Treatment-Emergent Adverse Events (TEAEs)

\begin{tabular}{|c|c|c|c|}
\hline \multirow[t]{3}{*}{ Adverse Events MedDRA Preferred Term } & $\begin{array}{l}\text { Elderly } \\
(\geq 65)\end{array}$ & $\begin{array}{l}\text { Non-Elderly } \\
(<65)\end{array}$ & Total \\
\hline & $(\mathbf{N}=33)$ & $(N=175)$ & $(N=208)$ \\
\hline & n (\%) & n (\%) & n (\%) \\
\hline At least one TEAE & $32(97.0)$ & $144(82.3)$ & I $76(84.6)$ \\
\hline Serious TEAEs & $2(6.1)$ & $24(13.7)$ & $26(12.5)$ \\
\hline TEAE leading to treatment discontinuation & $3(9.1)$ & $23(13.1)$ & $26(12.5)$ \\
\hline TEAE leading to dose reduction & $9(27.3)$ & $4 I(23.4)$ & $50(24.0)$ \\
\hline \multicolumn{4}{|l|}{ TEAEs occurring in $\geq 10 \%$ of patients in any group } \\
\hline Nasopharyngitis & $10(30.3)$ & $49(28.0)$ & $59(28.4)$ \\
\hline Schizophrenia & $9(27.3)$ & $38(21.7)$ & $47(22.6)$ \\
\hline Extrapyramidal disorder & $6(18.2)$ & $6(3.4)$ & $12(5.8)$ \\
\hline Contusion & $5(15.2)$ & $3(1.7)$ & $8(3.8)$ \\
\hline Influenza & $4(12.1)$ & $6(3.4)$ & $10(4.8)$ \\
\hline Insomnia & $4(12.1)$ & $19(10.9)$ & 23 (II.I) \\
\hline Headache & $2(6.1)$ & 20 (II.4) & $22(10.6)$ \\
\hline Akathisia & I (3.0) & $19(10.9)$ & $20(9.6)$ \\
\hline
\end{tabular}

Note: TEAEs were coded using the Medical Dictionary for Regulatory Activities (version 16.0).

Kaplan-Meier curves were constructed to display the percentages of patients who continued the trial (Figure 2 ). The most common reason for discontinuation was withdrawal of consent $(24.2 \%$ in elderly patients and $25.7 \%$ in non-elderly patients). The mean (standard deviation, SD) duration of treatment of brexpiprazole was 263.6 (156.4) days in elderly patients and 249.4 (160.0) days in non-elderly patients. The mean (SD) daily dose of brexpiprazole at week 56 was 3.0 (1.1) $\mathrm{mg} /$ day in elderly patients $(\mathrm{n}=14)$ and 3.2 (1.0) $\mathrm{mg} /$ day in non-elderly patients $(\mathrm{n}=64)$.

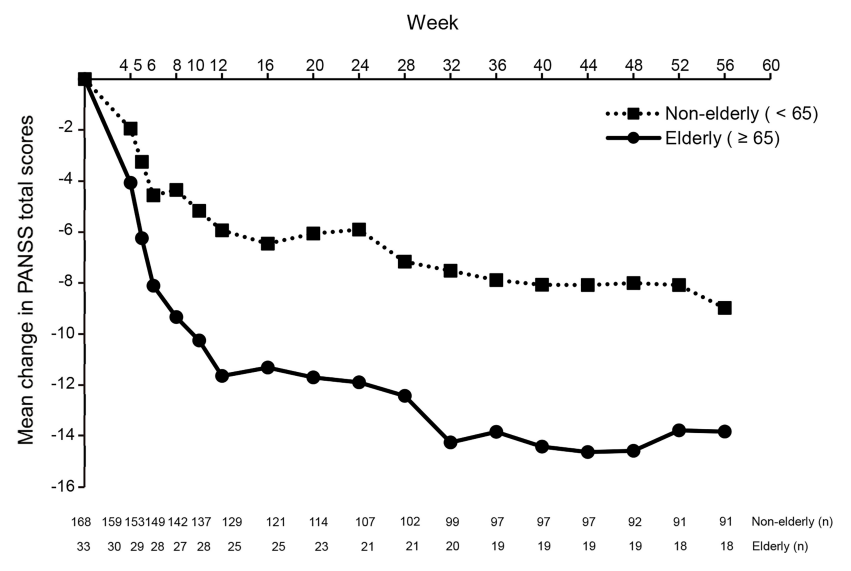

Figure 3 Mean change in PANSS total scores from baseline (observed cases).

\section{Efficacy}

Efficacy was assessed in 201 patients (33 elderly and 168 non-elderly). Mean change in the PANSS total score decreased sharply during the first $4-12$ weeks in elderly patients and during the first 4-6 weeks in non-elderly patients, and thereafter scores remained stable throughout the rest of the open-label period; mean change in PANSS total score from baseline to week 56 was -13.8 and -9.0 in elderly and non-elderly patients, respectively (Figure 3). Response rate at week 56 was $38.9 \%$ and $28.6 \%$ for elderly and non-elderly patients, respectively. The mean change in CGI-S score was -0.44 and -0.32 in elderly and non-elderly patients, respectively.

\section{Safety}

Safety was assessed in 208 patients (33 elderly and 175 non-elderly). Overall, 176 patients $(84.6 \%)$ experienced at least one TEAE, 32 (97.0\%) were elderly and 144 (82.3\%) were non-elderly patients (Table 2 and Supplemental Table

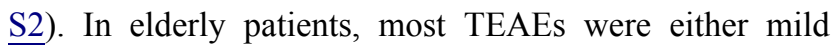
$(75.8 \%)$ or moderate $(18.2 \%)$ in severity, while the corresponding percentages in non-elderly patients were $56.6 \%$ and $20.6 \%$, respectively. Serious TEAEs were observed in 2 elderly patients $(6.1 \%)$ and 24 non-elderly patients $(13.7 \%)$. TEAEs leading to treatment discontinuation 
Table 3 Mean Change (SD) from Baseline to Last Visit in Extrapyramidal Rating Scale Scores

\begin{tabular}{|l|l|l|}
\hline Extrapyramidal Rating Scale Scores & $\begin{array}{l}\text { Elderly } \\
(\geq 65)\end{array}$ & $\begin{array}{l}\text { Non-Elderly } \\
(<65)\end{array}$ \\
\hline $\begin{array}{l}\text { Drug Induced Extrapyramidal Symptoms Scale Total score } \\
\text { Baseline, mean } \pm \text { SD (n) } \\
\text { Change at week 56, mean } \pm \text { SD (n) }\end{array}$ & $\begin{array}{l}2.76 \pm 4.09(33) \\
-0.44 \pm 2.68(18)\end{array}$ & $\begin{array}{l}1.10 \pm 2.19(175) \\
-0.26 \pm 1.98(91)\end{array}$ \\
\hline $\begin{array}{l}\text { Barnes Akathisia Rating Scale Global Score } \\
\text { Baseline, mean } \pm \text { SD (n) } \\
\text { Change at week 56, mean } \pm \text { SD (n) }\end{array}$ & $0.12 \pm 0.55(33)$ & $0.17 \pm 0.50(175)$ \\
\hline $\begin{array}{l}\text { Abnormal Involuntary Movement Scale Total Score } \\
\text { Baseline, mean } \pm \text { SD (n) } \\
\text { Change at week 56, mean } \pm \text { SD (n) }\end{array}$ & $-0.11 \pm 0.47(18)$ & $-0.08 \pm 0.34(91)$ \\
\hline
\end{tabular}

Abbreviation:SD, standard deviation.

occurred in 3 elderly patients $(9.1 \%)$ and 23 non-elderly patients (13.1\%). TEAEs leading to dose reduction occurred in 9 elderly patients $(27.3 \%)$ and 41 non-elderly patients (23.4\%). No deaths occurred during this study.

Table 2 shows TEAEs occurring in at least $10 \%$ of patients in either of the 2 groups. The most frequent TEAEs were nasopharyngitis and schizophrenia (specifically, worsening of schizophrenia), which were observed in $10(30.3 \%)$ and $9(27.3 \%)$ elderly patients, respectively. Comparable results were observed in non-elderly patients [49 (28.0\%) and $38(21.7 \%)$, respectively].

In terms of EPS related scores, no clinically meaningful changes were observed in either age group (Table 3). In the clinical laboratory tests, no elderly patients experienced the TEAE of "increased prolactin levels" or "decreased prolactin levels", while in non-elderly patients, $7(4.0 \%)$ and $1(0.6 \%)$ experienced mild "increased prolactin levels" and "decreased prolactin levels", respectively. The mean (SD) change in serum prolactin levels from baseline to week 56 was $-24.8(28.8) \mathrm{ng} / \mathrm{mL}$ in male elderly patients and $-21.6(29.4) \mathrm{ng} / \mathrm{mL}$ in female elderly patients. The corresponding figures for non-elderly patients were $-5.6(18.5) \mathrm{ng} / \mathrm{mL}$ in males and -8.9 (59.4) $\mathrm{ng} / \mathrm{mL}$ in females. A mild increase or decrease in body weight was observed in $1(3.0 \%)$ elderly patient each, while the corresponding figures for non-elderly patients were $10(5.7 \%)$ and $1(0.6 \%)$, respectively. The mean (SD) change in body weight from baseline to last visit and week 56 was $-0.1(4.3) \mathrm{kg}$ and $1.3(5.1) \mathrm{kg}$ in elderly patients, and $0.0(4.4) \mathrm{kg}$ and $0.51(5.0) \mathrm{kg}$ in nonelderly patients, respectively. One (3.0\%) elderly and 2 (1.1\%) non-elderly patients experienced mild "QTc interval prolongation" as a TEAE. Mean (SD) changes in the
QTc interval (Bazett's formula) from baseline to week 56 and the mean (SD) QTc interval (Bazett's formula) at week 56 were $1.1(20.0) \mathrm{msec}$ and 421.1 (25.6) msec in elderly patients, and -1.2 (20.0) msec and 415.7 (21.6) msec in non-elderly patients, respectively.

\section{Discussion}

This is a report on the long-term efficacy and safety of brexpiprazole in elderly patients with schizophrenia. This subgroup analysis based on age groups $(\geq 65$ years or $<65$ years) demonstrated that improvements in efficacy (change in the PANSS total score) were maintained and brexpiprazole was generally well tolerated in elderly Japanese patients with schizophrenia. ${ }^{13}$ Most participants were de novo outpatients, and the symptoms of elderly patients might have been slightly more severe than those of non-elderly patients because of higher dosages of prior antipsychotics and higher PANSS total scores at baseline (Table 1). The completion rates at week 56 were similar between the age groups (54.5\% in elderly and $52.0 \%$ in non-elderly patients; Figures 1 and 2 ). These results are consistent with the completion rate of $47.4 \%$ in another long-term trial in non-elderly patients with schizophrenia. ${ }^{12}$ These outcomes suggest that the ability of elderly patients to continue treatment with brexpiprazole is on a par with that of non-elderly patients. There were no significant differences in the mean daily dosage and treatment duration of brexpiprazole between elderly and nonelderly patients.

Based on the mean change in PANSS total score (Figure 3) and the response rate, long-term treatment with brexpiprazole was equally effective in both age groups, similar to the results obtained in another openlabel long-term study. ${ }^{12}$ The therapeutic effects were 
maintained during the 56-week treatment including the 4-week switching period. Moreover, those changes in elderly patients appeared somewhat larger than those seen in non-elderly patients though the sample size was small. Because the PANSS total score at baseline was higher in elderly than that in non-elderly patients (75.4 vs 68.9), this might have affected the mean change in PANSS total score at week 56. Even if these issues are taken into account, the results obtained in this study suggest that brexpiprazole should have a good efficacy profile in the long-term treatment of elderly patients with schizophrenia.

The incidence rates of TEAEs in elderly patients were slightly higher than those in non-elderly patients; however, most TEAEs were mild or moderate in severity and the incidence rates of serious TEAEs and TEAEs leading to treatment of discontinuation in elderly patients were numerically lower than those in non-elderly patients (Table 2 and Supplemental Table S2). The incidence of nasopharyngitis, contusion and influenza was higher in elderly patients, but these TEAEs were not potentially causally related to brexpiprazole. These results indicate that there was no tendency towards TEAEs being more severe or common in elderly patients and there were no major safety concerns.

The most common TEAEs were nasopharyngitis and worsening of schizophrenia in both groups. These had previously been observed in other long-term trials. ${ }^{10,12}$ Generally, EPS, hyperprolactinemia, weight gain and QTc prolongation are of concern when elderly patients with schizophrenia receive long-term treatment with antipsychotics. ${ }^{14}$ The incidence of EPS in elderly patients was higher than that in non-elderly patients; however, the severity of EPS was mild in all elderly patients and no clinically important changes in EPS scores including DIEPSS, AIMS and BARS scores, were observed in either elderly or non-elderly patients (Table 3 ). It is known that hyperprolactinemia is associated with a reduction in bone mineral density and osteoporosis/osteoporotic fractures, as well as sexual dysfunction, gynecomastia and amenorrhea. ${ }^{15}$ In this study treatment with brexpiprazole did not increase mean prolactin levels in elderly patients. This suggests that the risk of osteoporosis/osteoporotic fractures is probably low. Body weight increase and QTc prolongation were observed in some patients, as reported in previous open-label long-term studies, ${ }^{11,12,16}$ but the occurrence rates were similar between the age groups. The mean increase in body weight was $1.3 \mathrm{~kg}$ in elderly patients and $0.51 \mathrm{~kg}$ in non-elderly patients who completed the 56-week study. It is surmised that this was partly due to the lower average body weight of the elderly patients at the baseline $(55.0 \mathrm{~kg})$, while that of the nonelderly patients was $66.7 \mathrm{~kg}$ (Table 1). In addition, we compared mean changes in metabolic parameters from baseline to week 56, but no significant differences were observed between age groups (Supplementary Table S3). Overall, these results showed that brexpiprazole had an acceptable safety profile with favorable tolerability in the long-term treatment of schizophrenia in elderly patients as well as non-elderly patients. Considering the safety profiles observed in previous clinical trials as well as in the current study, despite the requirement for careful adjustments to dosing based on observation of the condition of elderly patients, it is believed that most elderly patients could be treated with doses of brexpiprazole similar to those administered to non-elderly patients.

This study has several limitations. There are some biases associated with the study's design. One is the small sample size, especially for the elderly patients, which made it unfeasible to conduct statistical tests to evaluate the outcome measures. The enrolled patients were required to meet inclusion and exclusion criteria, and this means that the study population may not be representative of a real-world clinical setting. It may therefore be necessary to collect such data using a database. The other is the lack of a placebo control group. As was shown in the previously reported randomized double-blind placebo-controlled studies, in which the average treatment change associated with placebo treatment in antipsychotic trials has been increasing, ${ }^{17,18}$ the placebo effect would be a confounding factor that may need to be considered when interpreting the results of this study.

\section{Conclusion}

There have only been a few reports in which treatment outcomes with brexpiprazole have been assessed in elderly patients with schizophrenia. This study confirmed the favorable long-term efficacy, safety and tolerability of brexpiprazole in elderly Japanese patients with schizophrenia, mirroring the results obtained in previous studies of adult patients. Overall, this post hoc analysis suggests that brexpiprazole could be a promising option in the long-term treatment of elderly patients with schizophrenia.

\section{Data Sharing Statement}

The data that support the findings of this study are proprietary in nature and are not publicly available. However, 
the data are available if a reasonable request is made to the corresponding author upon reasonable request.

\section{Acknowledgments}

We gratefully acknowledge the support of the patients and all investigators at each study site through their participation in this study. Data analysis was supported by A2 Healthcare Corporation (Osaka, Japan); medical writing support was provided by Kazuo Nakajima, PhD (SunFlare Co., Ltd., Tokyo, Japan), and funding was provided by Otsuka Pharmaceutical Co., Ltd.

\section{Author Contributions}

All authors made a significant contribution to the work reported, whether that is in the conception, study design, execution, acquisition of data, analysis and interpretation, or in all these areas; took part in drafting, revising or critically reviewing the article; gave final approval of the version to be published; have agreed on the journal to which the article has been submitted; and agree to be accountable for all aspects of the work.

\section{Disclosure}

This study was funded by Otsuka Pharmaceutical Co., Ltd. Dr. Inada has received speaker's honoraria from Eisai, Eli Lilly Japan, Janssen, Meiji Seika Pharma, Mitsubishi Tanabe Pharma, Mochida, MSD, Novartis, Otsuka pharmaceutical Co., Ltd., Shionogi, Sumitomo Dainippon Pharma, and Yoshitomiyakuhin, and research grants from Japan Agency for Medical Research and Development (AMED), Ministry of Health, Labour and Welfare, Mitsubishi Tanabe Pharma, MSD, and National Center of Neurology and Psychiatry (NCNP). Dr. Ishigooka has received speaker's honoraria from Astellas, Alfresa, Novartis, Otsuka pharmaceutical Co., Ltd, Sumitomo Dainippon Pharma, Eisai, Eli Lilly Japan, and Takeda pharmaceutical Co., Ltd. Ms. Yamada, Mr. Akiyoshi, Mr. Kojima and Mr. Iwashita are employees of Otsuka Pharmaceutical Co., Ltd. The authors report no other conflicts of interest in this work.

\section{References}

1. GBD. 2017 disease and injury incidence and prevalence collaborators. Global, regional, and national incidence, prevalence, and years lived with disability for 354 diseases and injuries for 195 countries and territories, 1990-2017: a systematic analysis for the global burden of disease study 2017. Lancet. 2018;392(10159):1789-1858.
2. Rochon PA. Drug prescribing for older adults [Internet]. [updated Aug 15, 2019; cited May 12, 2020]. Available from: https://www. uptodate.com/contents/drug-prescribing-for-older-adults.

3. Citrome L, Stensbøl TB, Maeda K. The preclinical profile of brexpiprazole: what is its clinical relevance for the treatment of psychiatric disorders? Expert Rev Neurother. 2015;15(10):1219-1229. doi:10.1586/14737175.2015.1086269

4. Bruijnzeel D, Tandon R. Spotlight on brexpiprazole and its potential in the treatment of schizophrenia and as adjunctive therapy for the treatment of major depression. Drug Des Devel Ther. 2016;10:1641-1647.

5. Maeda K, Sugino H, Akazawa H, et al. Brexpiprazole I: in vitro and in vivo characterization of a novel serotonin-dopamine activity modulator. $J$ Pharmacol Exp Ther. 2014;350(3):589-604. doi:10.1124/jpet.114.213793

6. Maeda K, Lerdrup L, Sugino $H$, et al. Brexpiprazole II: antipsychotic-like and procognitive effects of a novel serotonin-dopamine activity modulator. J Pharmacol Exp Ther. 2014;350(3):605-614. doi:10.1124/jpet.114.213819

7. Correll CU, Skuban A, Ouyang J, et al. Efficacy and safety of brexpiprazole for the treatment of acute schizophrenia: a 6-week randomized, double-blind, placebo-controlled trial. Am J Psychiatry. 2015;172(9):870-880. doi:10.1176/appi.ajp.2015.14101275

8. Kane JM, Skuban A, Ouyang J, et al. A multicenter, randomized, double-blind, controlled Phase 3 trial of fixed-dose brexpiprazole for the treatment of adults with acute schizophrenia. Schizophr Res. 2015;164(1-3):127-135. doi:10.1016/j.schres.2015.01.038

9. Ishigooka J, Iwashita S, Tadori Y. Efficacy and safety of brexpiprazole for the treatment of acute schizophrenia in Japan: a 6-week, randomized, double-blind, placebo-controlled study. Psychiatry Clin Neurosci. 2018;72(9):692-700. doi:10.1111/pcn.12682

10. Fleischhacker WW, Hobart M, Ouyang J, et al. Efficacy and safety of brexpiprazole (OPC-34712) as maintenance treatment in adults with schizophrenia: a randomized, double-blind, placebo-controlled study. Int J Neuropsychopharmacol. 2017;20(1):11-21.

11. Kane JM, Skuban A, Hobart M, et al. Overview of short- and long-term tolerability and safety of brexpiprazole in patients with schizophrenia. Schizophr Res. 2016;174(1-3):93-98. doi:10.1016/j. schres.2016.04.013

12. Forbes A, Hobart M, Ouyang J, et al. A long-term, open-label study to evaluate the safety and tolerability of brexpiprazole as maintenance treatment in adults with schizophrenia. Int J Neuropsychopharmacol. 2018;21(5):433-441. doi:10.1093/ ijnp/pyy002

13. Ishigooka J, Iwashita $\mathrm{S}$, Tadori Y. Long-term safety and effectiveness of brexpiprazole in Japanese patients with schizophrenia: a 52-week, open-label study. Psychiatry Clin Neurosci. 2018;72(6):445-453.

14. Maixner SM, Mellow AM, Tandon R. The efficacy, safety, and tolerability of antipsychotics in the elderly. $J$ Clin Psychiatry. 1999;60(Suppl 8):29-41.

15. De Hert M, Detraux J, Stubbs B. Relationship between antipsychotic medication, serum prolactin levels and osteoporosis/osteoporotic fractures in patients with schizophrenia: a critical literature review. Expert Opin Drug Saf. 2016;15(6):809-823. doi:10.1517/ 14740338.2016.1167873

16. Weiss C, Weiller E, Baker RA, et al. The effects of brexpiprazole and aripiprazole on body weight as monotherapy in patients with schizophrenia and as adjunctive treatment in patients with major depressive disorder: an analysis of short-term and long-term studies. Int Clin Psychopharmacol. 2018;33(5):255-260.

17. Rutherford BR, Pott E, Tandler JM, et al. Placebo response in antipsychotic clinical trials: a meta-analysis. JAMA Psychiatry. 2014;71 (12):1409-1421. doi:10.1001/jamapsychiatry.2014.1319

18. Weimer K, Colloca L, Enck P. Age and sex as moderators of the placebo response- an evaluation of systematic reviews and metaanalyses across medicine. Gerontology. 2015;61(2):97-108. 


\section{Publish your work in this journal}

Neuropsychiatric Disease and Treatment is an international, peerreviewed journal of clinical therapeutics and pharmacology focusing on concise rapid reporting of clinical or pre-clinical studies on a range of neuropsychiatric and neurological disorders. This journal is indexed on PubMed Central, the 'PsycINFO' database and CAS, and is the official journal of The International Neuropsychiatric Association (INA). The manuscript management system is completely online and includes a very quick and fair peer-review system, which is all easy to use. Visit http://www.dovepress.com/testimonials.php to read real quotes from published authors.

Submit your manuscript here: https://www.dovepress.com/neuropsychiatric-disease-and-treatment-journal 